\title{
A NEW LIMIT ON SCALAR AND VECTOR CONTRIBUTIONS TO GRAVITY
}

\author{
G.W. FORD and D.J. HEGYI \\ Department of Physics, The University of Michigan, Ann Arbor, MI 48109-1120, USA
}

Received 18 November 1988

\begin{abstract}
Macroscopic effects of theories of quantum gravity containing scalar, vector, and tensor component sin proportions $c_{\mathrm{S}}: c_{\mathrm{V}}: c_{\mathrm{T}}$ are considered. Assuming zero mass fields, the classical tests of general relativity are found to imply, to $90 \%$ confidence, $c_{\mathrm{T}}=1 \pm 0.003$ and $\left|c_{V}-c_{S}\right|<0.003$. For the binary pulsar PSR $1913+16$, the calculated gravitational radiation is compared with the measured emission and found to imply $0 \leqslant c_{\mathrm{S}}<0.12$. These results, which are independent of any assumption about differences between matter-matter and matter-antimatter interactions, place meaningful constraints on local supersymmetric theories of gravity.
\end{abstract}

There has been much recent interest in the possibility that quantum theories of gravity might imply the existence of scalar (S) and vector (V) components of the gravitational field in addition to a tensor ( $T$ ) component corresponding to the familiar Einstein theory. These additional fields, which appear in a natural way in local supersymmetric theories of gravity [1], may be expected to have observable consequences at the macroscopic level $[2,3]$. In particular, if these were zero mass fields then ordinary newtonian gravity would be the sum of scalar, vector and tensor contributions. For interactions between ordinary matter, the vector component of the force in this version of newtonian gravity would be repulsive and tend to cancel the attractive scalar and tensor components. For gravitational interaction between matter and antimatter the interesting suggestion has been made that the vector force would be attractive and so would not cancel and could lead to large observable effects. This is one of the motives for an experiment at the LEAR facility at CERN in which the acceleration due to gravity is to be measured for antiprotons $[3,4]$. Here we point out that meaningful limits on these scalar and vector components can be set using measurements of gravitational wave emission in the binary pulsar PSR $1913+16$. An important feature of these limits is that they are independent of any assumption about the nature of the matter-antimatter gravitational interaction.

We begin by introducing a phenomenological field theory of macroscopic gravitation in which a collection of point-like particles of inertial mass $m_{j}$ interact through a gravitational field which is a mixture of scalar, vector and tensor fields in proportions $c_{\mathrm{S}}: c_{\mathrm{V}}: c_{\mathrm{T}}$. In order to be specific and to quantify our remarks, we exhibit the lagrangian density for this theory, which is of the form

$$
\mathscr{L}=\mathscr{L}_{\mathrm{S}}+\mathscr{L}_{\mathrm{V}}+\mathscr{L}_{\mathrm{T}}
$$

where (with the metric $g_{00}=-g_{11}=-g_{22}=-g_{33}=1$ )

$$
\begin{aligned}
\mathscr{L}_{\mathrm{S}} & =\frac{1}{8 \pi G c_{\mathrm{S}}} g^{\kappa \lambda} \frac{\partial \phi}{\partial x^{\kappa}} \frac{\partial \phi}{\partial x^{\lambda}} \\
& -\sum_{j} m_{j}\left(1-\frac{v_{j}^{2}}{c^{2}}\right)^{1 / 2} \phi\left(\boldsymbol{r}_{j}, t\right) \delta\left(\boldsymbol{r}-\boldsymbol{r}_{j}\right), \\
\mathscr{L}_{\mathrm{V}} & =-\frac{1}{8 \pi G c_{\mathrm{V}}}\left(g^{\kappa \lambda} \frac{\partial \phi^{\mu}}{\partial x^{\kappa}} \frac{\partial \phi_{\mu}}{\partial x^{\lambda}}-\frac{\partial \phi^{\mu}}{\partial x^{\nu}} \frac{\partial \phi^{\nu}}{\partial x^{\mu}}\right) \\
& -\frac{1}{c} \sum_{j} m_{j} \phi_{\mu}\left(\boldsymbol{r}_{j}, t\right) \frac{\mathrm{d} x_{j}^{\mu}}{\mathrm{d} t} \delta\left(\boldsymbol{r}-\boldsymbol{r}_{j}\right), \\
\mathscr{L}_{\mathrm{T}} & =\frac{1}{16 \pi G c_{\mathrm{T}}}\left(g^{\kappa \lambda} \frac{\partial \phi_{\nu}^{\mu}}{\partial x^{\kappa}} \frac{\partial \phi_{\mu}^{\nu}}{\partial x^{\lambda}}-2 \frac{\partial \phi_{\mu}^{\kappa}}{\partial x^{\kappa}} \frac{\partial \phi^{\mu \lambda}}{\partial x^{\lambda}}\right. \\
& \left.+2 \frac{\partial \phi^{\kappa \lambda}}{\partial x^{\kappa}} \frac{\partial \phi_{\mu}^{\mu}}{\partial x^{\lambda}}-g^{\kappa \lambda} \frac{\partial \phi_{\mu}^{\mu}}{\partial x^{\kappa}} \frac{\partial \phi_{\nu}^{\nu}}{\partial x^{\lambda}}\right)-\sum_{j} m_{j} c^{2}\left(1+\frac{v_{j}^{2}}{c^{2}}\right. \\
& \left.+\frac{2}{c^{4}} \phi_{\mu \nu}\left(\boldsymbol{r}_{j}, t\right) \frac{\mathrm{d} x_{j}^{\mu}}{\mathrm{d} t} \frac{\mathrm{d} x_{j}^{\nu}}{\mathrm{d} t}\right)^{1 / 2} \delta\left(\boldsymbol{r}-\boldsymbol{r}_{j}\right) .
\end{aligned}
$$


Here the tensor lagrangian density corresponds to a tensor field theory equivalent to the Einstein theory through the post-newtonian terms. We are aware of the difficulty in conceiving how such a macroscopic theory could arise from our current understanding of the fundamental interactions. We emphasize, however, that this is the most general field theory consistent with (special) relativistic invariance and the newtonian form of non-relativistic gravitation, and, therefore, any macroscopic theory of scalar-vectortensor gravitation must have this form. Before discussing gravitational radiation we consider the constraints set upon the positive constants $c_{\mathrm{S}}, c_{\mathrm{V}}$ and $c_{\mathrm{T}}$ by the definition of the gravitational constant $G$ and by the classical tests of general relativity. For the theory described by the above lagrangian density the nonrelativistic gravitational potential arising from a static central mass $M$ is

$\phi_{\mathrm{NR}}=\left(c_{\mathrm{S}}-c_{\mathrm{V}}+c_{\mathrm{T}}\right) \frac{M G}{r}$.

The definition of $G$ or, if one prefers, the Eötvos experiments require that this must be of the form of the newtonian potential. Therefore we must have the constraint

$c_{\mathrm{S}}-c_{\mathrm{V}}+c_{\mathrm{T}}=1$.

The bending of light calculated in this theory is given by

$$
\Delta \vartheta=c_{\mathrm{T}} \frac{4 M G}{c^{2} r},
$$

the scalar and vector fields making no contribution. The most recent experimental results on light bending [5], and on the equivalent radar ranging time delay $[6,7]$, are consistent with

$c_{\mathrm{T}}=1 \pm 0.002$.

The precession of the perihelion calculated in this theory is

$\dot{\omega}=\left(-\frac{1}{6} c_{\mathrm{S}}+\frac{1}{6} c_{\mathrm{V}}+c_{\mathrm{T}}\right) \frac{3(M G)^{3 / 2}}{c^{2} a^{5 / 2}\left(1-e^{2}\right)}$.

The observations of Mercury are consistent with $[8,9]$

$$
-\frac{1}{6} c_{\mathrm{S}}+\frac{1}{6} c_{\mathrm{V}}+c_{\mathrm{T}}=1 \pm 0.01
$$

Combining (4), (6) and (8) we see that the classical tests are consistent with $c_{\mathrm{T}}=1$ and $c_{\mathrm{S}}=c_{\mathrm{V}}$ at the level of $0.2 \%$, but there is no constraint on the common value of $c_{\mathrm{S}}$ and $c_{\mathrm{V}}$. This is the interesting possibility suggested by Macrae and Riegert [2].

We consider now gravitational radiation by a binary system in the theory described by the lagrangian density (1). The field equation for the scalar field is [10]

$\square \phi=4 \pi G c_{\mathrm{S}} \rho(r, t)$,

where the source density is

$\rho(\boldsymbol{r}, t)=\sum_{j} m_{j}\left(1-\frac{v_{j}(t)^{2}}{c^{2}}\right)^{1 / 2} \delta\left(\boldsymbol{r}-\boldsymbol{r}_{j}(t)\right)$.

Here the sum is over the two stars in the binary which, on account of (4), are in a Kepler orbit. Far from the source the solution of the field equation has the form

$\phi(\boldsymbol{r}, t) \approx \frac{G c_{\mathrm{S}}}{r} \int \mathrm{d} \boldsymbol{r}^{\prime} \rho\left(\boldsymbol{r}^{\prime}, t-r / c-\hat{\boldsymbol{n}} \cdot \boldsymbol{r}^{\prime} / c\right)$,

where $\hat{n}=\boldsymbol{r} / r$. As usually stated, the condition for the validity of the multipole expansion is that the wavelength of the emitted radiation is long compared with the size of the source. For a Kepler orbit with semimajor axis $a$ and period $T$, this becomes

$a / c T \ll 1$.

For the binary pulsar PSR $1913+16, a / c T=2.33$ $\times 10^{-4}$, so this is comfortably satisfied [11]. Also, this is the condition that typical velocities in the source are small compared with the velocity of light, so the Kepler orbit is non-relativistic. The result is that we may expand the integrand in (11) in powers of the small time $\hat{\boldsymbol{n}} \cdot \boldsymbol{r}^{\prime} / \mathcal{c}$, and within the expression (10) we may expand in powers of $v_{j}^{2} / c^{2}$ to get

$$
\begin{gathered}
\phi(\boldsymbol{r}, t)=\frac{G c_{\mathrm{S}}}{r} \sum_{j} m_{j}\left\{1-\frac{1}{c} \frac{\mathrm{d}}{\mathrm{d} t} \hat{\boldsymbol{n}} \cdot \boldsymbol{r}_{j}\left(t-\frac{r}{c}\right)\right. \\
\left.+\frac{1}{2 \mathrm{c}^{2}}\left[\frac{\mathrm{d}^{2}}{\mathrm{~d} t^{2}} \hat{\boldsymbol{n}} \cdot \boldsymbol{r}_{j}\left(t-\frac{r}{c}\right)^{2}-v_{j}\left(t-\frac{r}{c}\right)^{2}\right]\right\} .
\end{gathered}
$$

But the total mass,

$M=\sum_{j} m_{j}=m_{1}+m_{2}$,

as well as the coordinate of the center of mass, 
$\boldsymbol{R}=\frac{1}{M} \sum_{j} m_{j} \boldsymbol{r}_{j}(t)$,

are constants of the non-relativistic Kepler motion of the binary. There are relativistic corrections of order $1 / c^{2}$ to the center-of-mass motion, but these are of higher order and may be neglected. Therefore, to the accuracy we need, the first two terms within the square bracket in (13) make no contribution at finite frequency. In the remaining terms we introduce relative coordinates, writing

$\boldsymbol{r}_{1}=\boldsymbol{R}+\frac{m_{2}}{M} \boldsymbol{\xi}, \quad \boldsymbol{r}_{2}=\boldsymbol{R}-\frac{m_{1}}{M} \boldsymbol{\xi}$,

to get

$\phi(r, t)=\frac{\mu G c_{\mathrm{S}}}{2 r c^{2}}\left[\frac{\mathrm{d}^{2}}{\mathrm{~d} t^{2}} \hat{\boldsymbol{n}} \cdot \boldsymbol{\xi}\left(t-\frac{r}{c}\right)^{2}-v\left(t-\frac{r}{c}\right)^{2}\right]$,

where $\mu=m_{1} m_{2} / M$ is the reduced mass and $v$ is the relative velocity.

The radiated power per unit solid angle is

$\frac{\mathrm{d} P}{\mathrm{~d} \Omega}=\lim _{r \rightarrow \infty} r^{2} \hat{n} \cdot S$,

where $S$ is the energy flux density and is given by [10]

$S=-\frac{1}{4 \pi G c_{\mathrm{S}}} \frac{\partial \phi}{\partial t} \nabla \phi \approx \frac{1}{4 \pi G c_{\mathrm{S}} c}\left(\frac{\partial \phi}{\partial t}\right)^{2} \hat{n}$.

Using the expression (17) for $\phi(r, t)$ we find

$\frac{\mathrm{d} P}{\mathrm{~d} \Omega}=\frac{\mu^{2} G c_{\mathrm{S}}}{16 \pi c^{5}}\left(\frac{\mathrm{d}^{3}}{\mathrm{~d} t^{3}} \hat{n} \cdot \xi^{2}-\frac{\mathrm{d}}{\mathrm{d} t} v^{2}\right)^{2}$.

We can work out the time derivatives in this expression using the equations of Kepler motion:

$\frac{\mathrm{d} \xi}{\mathrm{d} t}=v, \quad \frac{\mathrm{d} \boldsymbol{v}}{\mathrm{d} t}=-M G \frac{\boldsymbol{\xi}}{\xi^{3}}$,

where, again, we can use the constraint (4) for the non-relativistic Kepler motion. The result is

$$
\begin{aligned}
\frac{\mathrm{d} P}{\mathrm{~d} \Omega} & =\frac{(\mu M G)^{2} G c_{\mathrm{S}}}{4 \pi c^{5}} \\
& \times\left(4 \frac{\boldsymbol{n} \cdot \boldsymbol{\xi} \hat{\boldsymbol{n}} \cdot \boldsymbol{v}}{\xi^{3}}-3 \frac{(\hat{\boldsymbol{n}} \cdot \boldsymbol{\xi})^{2} \boldsymbol{\xi} \cdot \boldsymbol{v}}{\xi^{5}}-\frac{\boldsymbol{\xi} \cdot \boldsymbol{v}}{\boldsymbol{\xi}^{3}}\right)^{2} .
\end{aligned}
$$

Integrating over directions of $\hat{\boldsymbol{n}}$, using $\overline{\hat{n}_{i} \hat{n}_{j}}=\frac{1}{3} \delta_{i j}$,

$\overline{\hat{n}_{i}} \hat{n}_{j} \hat{n}_{k} \hat{n}_{l}=\frac{1}{15}\left(\delta_{i j} \delta_{k l}+\delta_{i k} \delta_{j l}+\delta_{i l} \delta_{j k}\right)$,

we find for the total instantaneous power radiated

$P=\frac{8(\mu M G)^{2} G c_{\mathrm{S}}}{15 c^{5}}\left(2 \frac{v^{2}}{\xi^{4}}-\frac{(\xi \cdot v)^{2}}{\xi^{6}}\right)$.

To find the time-average power we average over a period of the Kepler motion, using the Kepler orbital equations:

$\xi=a(1-e \cos u), \quad \frac{2 \pi t}{T}=u-e \cos u$,

$\frac{2 \pi}{T}=\sqrt{\frac{M G}{a^{3}}}, \quad v^{2}=\left(\frac{2 \pi a}{T}\right)^{2} \frac{1+e \cos u}{1-e \cos u}$

$\boldsymbol{\xi} \cdot \boldsymbol{v}=\frac{2 \pi a^{2}}{T} e \sin u$

The final result is the following expression for the time-average power radiated in the form of scalar fields:

$P_{\mathrm{S}}=c_{\mathrm{S}} \frac{2 \pi \mu^{2} G}{15 a T}\left(\frac{2 \pi a}{c T}\right)^{5} \frac{16+52 e^{2}+7 e^{4}}{\left(1-e^{2}\right)^{7 / 2}}$.

The derivation of the corresponding results for time-average power radiated in the form of vector and tensor fields is entirely similar. The two differ by only a constant factor,

$P_{\mathrm{V}}=\frac{c_{\mathrm{V}}}{4 c_{\mathrm{T}}} P_{\mathrm{T}}$,

and the result for $P_{\mathrm{T}}$ is in the literature [12]

$P_{\mathrm{T}}=c_{\mathrm{T}} \frac{2 \pi \mu^{2} G}{15 a T}\left(\frac{2 \pi a}{c T}\right)^{5} \frac{96+292 e^{2}+37 e^{4}}{\left(1-e^{2}\right)^{7 / 2}}$.

The total radiated power is the sum

$P=P_{\mathrm{S}}+P_{\mathrm{V}}+P_{\mathrm{T}}$.

We have given the derivation of this result, with some detail in the scalar case, because we wish to emphasize several important features. The first of these is that the power radiated in the form of scalar, vector and tensor fields is proportional, respectively, to the positive constants $c_{\mathrm{S}}, c_{\mathrm{V}}$ and $c_{\mathrm{T}}$ (not their squares, as one might naively think). Another feature is that there is no dipolar radiation of either scalar or vector 
fields. As we have seen, this is a consequence of the fact that the source is a pair of masses in a non-relativistic Kepler orbit, for which the total mass and the coordinate of the center of mass are constant. The last term in (13), the term containing a factor $v_{j}^{2}$, may be considered as a relativistic monopole term, but it is of the same order as the quadrupole terms.

The observations on the binary pulsar PSR $1913+16$ are consistent with [13]

$$
0.173 c_{\mathrm{S}}+0.250 c_{\mathrm{V}}+c_{\mathrm{T}}=1.00 \pm 0.04 \text {. }
$$

If we use the constraint (4) to eliminate $c_{\mathrm{V}}$ and use the value (6) of $c_{\mathrm{T}}$ set by the classical tests, this becomes

$c_{\mathrm{S}}=0.00 \pm 0.09$,

where, as in all the above, the quoted uncertainty represents one standard deviation. To transform this into an upper limit, we use the important constraint that $c_{\mathrm{S}}$ and $c_{\mathrm{V}}$ must be positive. The conclusion is that at the level of $90 \%$ confidence,

$0 \leqslant c_{\mathrm{S}}<0.12, \quad\left|c_{\mathrm{S}}-c_{\mathrm{V}}\right|<0.003$.

Before closing, we should remark upon the severe constraint placed upon the vector coupling for the $\mathrm{K}_{0^{-}}$ $\overline{\mathrm{K}}_{0}$ system [14]. It has been argued that, since the $\mathrm{K}$ meson consists of a bound quark-antiquark pair, the vector coupling might be zero for this system, though not for ordinary matter [2]. An important feature of the constraints we have obtained is that they are independent of any assumption about the difference between matter-matter and matter-antimatter gravitational interaction.

In summary, we have placed a new constraint on the possible contribution of scalar and vector components of gravity using the measurements of the power radiated by the binary pulsar PSR $1913+16$ combined with the constraints imposed by the classical tests of general relativity. This constraint is meaningful for theories of quantum gravity as well as for proposed experiments to measure these components by other means.

We thank M. Veltman for helpful discussions.

\section{References}

[1] C.K. Zachos, Phys. Lett. B 76 (1978) 329

[2] K.I. Macrae and R.J. Riegert, Nucl. Phys. B 244 (1984) 513.

[3] T. Goldman, R.J. Hughes and M.M. Nieto, Proc. Intern. Conf. on High energy physics (Berkeley, 1986).

[4] R.E. Brown, Proc. 2nd Conf. on the Intersections between particle physics and nuclear physics (Lake Louise, Canada, 1986).

[5] E.B. Fomolont and R.A. Sramek, Comm. Astro. 7 (1977) 19.

[6] D.L. Cain et al., Bull. Am. Astron. Soc. 10 (1978) 396.

[7] R.D. Reasenberg et al., Astorphys. J. 234 (1979) L219.

[8] G.M. Clemence, Rev. Mod. Phys. 19 (1947) 361.

[9] R.H. Dicke, Nature 202 (1964) 452.

[10] G. Wentzel, Quantum field theory (Interscience, New York, 1949).

[11] J.H. Taylor and J.M. Weisberg, Astrophys. J. 253 (1982) 908.

[12] P.C. Peters and J. Mathews, Phys. Rev. 131 (1963) 435.

[13] J.H. Taylor, Proc. 11 th Intern. Conf. on General relativity and gravitation (Cambridge U.P., Cambridge, 1986).

[14] M.L. Good, Phys. Rev. 121 (1961) 311. 